\title{
Probing IMF using nanodust measurements from inside Saturn's magnetosphere
}

\author{
H.-W. Hsu, ${ }^{1}$ K. C. Hansen, ${ }^{2}$ M. Horányi, ${ }^{1}$ S. Kempf, ${ }^{1}$ A. Mocker,,${ }^{1,3}$ \\ G. Moragas-Klostermeyer, ${ }^{3}$ F. Postberg, ${ }^{3,4}$ R. Srama, ${ }^{3}$ and B. Zieger ${ }^{5}$ \\ Received 13 May 2013; accepted 29 May 2013; published 18 June 2013.
}

[1] We present a new concept of monitoring the interplanetary magnetic field (IMF) by using in situ measurements of nanodust stream particles in Saturn's magnetosphere. We show that the nanodust detection pattern obtained inside the magnetosphere resembles those observed in interplanetary space and is associated with the solar wind compression regions. Our dust dynamics model reproduces the observed nanodust dynamical properties as well as the detection pattern, suggesting that the ejected stream particles can reenter Saturn's magnetosphere at certain occasions due to the dynamical influence from the time-varying IMF. This method provides information on the IMF direction and a rough estimation on the solar wind compression arrival time at Saturn. Such information can be useful for studies related to the solar wind-magnetosphere interactions, especially when the solar wind parameters are not directly available. Citation: Hsu, H.-W., K. C. Hansen, M. Horányi, S. Kempf, A. Mocker, G. Moragas-Klostermeyer, F. Postberg, R. Srama, and B. Zieger (2013), Probing IMF using nanodust measurements from inside Saturn's magnetosphere, Geophys. Res. Lett., 40, 2902-2906, doi:10.1002/grl.50604.

\section{Introduction}

[2] The dynamics of charged nanodust particles are of particular interest because of their intermediate charge-tomass ratio $\left(\sim 10^{3}\right.$ to $\left.10^{4} \mathrm{C} / \mathrm{kg}\right)$. Compared to those of nominal plasma ions (e.g., the charge-to-mass ratio of $\mathrm{O}^{+}$ is $\sim 6 \cdot 10^{6} \mathrm{C} / \mathrm{kg}$ ), the gyroradius of charged nanoparticles is fairly large but their dynamics are nonetheless electromagnetic force dominant. In contrast to thermal plasma particles, the fact that the dynamics of charged nanodust particles do not follow the guiding center approximation makes them a useful remote sensing tool for probing the variation of their electromagnetic environments.

[3] In this work, we demonstrate a new concept of using nanodust measurements inside the magnetosphere, as a remote sensing tool, to probe the interplanetary magnetic

\footnotetext{
${ }^{1}$ Laboratory for Atmospheric and Space Physics, University of Colorado, Boulder, Colorado, USA.

${ }^{2}$ Department of Atmospheric, Oceanic and Space Sciences, University of Michigan, Ann Arbor, Michigan, USA

${ }^{3}$ Institute of Space Systems, University of Stuttgart, Stuttgart, Germany.

${ }^{4}$ Geoscience Institute, Heidelberg University, Heidelberg, Germany.

${ }^{5}$ Center for Space Physics, Boston University, Boston, Massachusetts, USA.

Corresponding author: H.-W. Hsu, Laboratory for Atmospheric and Space Physics, University of Colorado Boulder 3665 Discovery Drive Boulder, Colorado 80303, CO, USA. (sean.hsu@lasp.colorado.edu)

(C)2013. American Geophysical Union. All Rights Reserved. 0094-8276/13/10.1002/grl.50604
}

field (IMF) properties. This concept is supported by the Cassini dust measurements at Saturn (section 2) as well as results from the Michigan Solar Wind Model (mSWiM) (section 3) [Zieger et al., 2008] and the nanodust dynamics simulation (section 4). The strength and limitation of this approach are summarized in section 5 .

\section{Fast Nanodust Particles From Saturn and Their Interactions With Solar Wind}

[4] After the Jovian system, the Saturnian system is the second identified source of fast nanodust in the solar system [Grün et al., 1993; Kempf et al., 2005]. Positively charged nanoparticles in Saturn's magnetosphere experience acceleration by the corotation electric field and escape the system as so-called "stream particles" (see Horányi et al. [1997], Horányi [2000], and Hsu et al. [2011b, 2012] for more details on the ejection process). Once in interplanetary space, the motion of stream particles is governed by the IMF and they will again be accelerated and eventually "picked up" by the magnetic and electric fields in the solar wind. Saturnian stream particles are found to have charge-tomass ratios ranging from 1000 to $20,000 \mathrm{C} / \mathrm{kg}(2$ to $8 \mathrm{~nm}$ in radius) and ejection speed around 50 to $200 \mathrm{~km} \mathrm{~s}^{-1}$ [Hsu et al., 2011b]. The dynamics of stream particles show more complexities than those of pickup ions (PUIs) due to their moderate charge-to-mass ratio. Their dynamical reactions to the changing IMF conditions in the vicinity of the source planet, where the pickup process takes place, are of particular interest here.

[5] Considering only electromagnetic forces from the IMF, the equation of motion of a charged particle with charge-to-mass ratio $q / m$ and velocity vector $\mathbf{v}$ is

$$
\dot{\mathbf{v}}=\frac{q}{m} \cdot\left[\mathbf{v} \times \mathbf{B}_{\mathrm{IMF}}+\mathbf{E}_{\mathbf{c}}\right],
$$

where $\mathbf{B}_{\mathrm{IMF}}$ is the IMF vector and $\mathbf{E}_{\mathbf{c}}=-v_{\mathrm{sw}} \times \mathbf{B}_{\mathbf{I M F}}$ is the convection electric field resulting from the solar wind flow carrying its "frozen-in" magnetic field. For PUIs, $\mathbf{E}_{\mathbf{c}}$ is the dominant term in equation (1) as their initial particle speed is slower than the solar wind speed $\left(\left|v_{\mathrm{sw}}\right| \sim 400 \mathrm{~km} \mathrm{~s}^{-1}\right)$ [Hill et al., 2004]. The dynamics of charged nanoparticles, on the other hand, are more complex as their speeds are comparable to the solar wind speed. Moreover, due to the difference in the charge-to-mass ratio, the gyroradius and gyroperiod of charged nanodust are much larger for PUIs. Considering an IMF strength of $0.2 \mathrm{nT}$, the gyroradius of a nanoparticle (with $q / m$ of $5000 \mathrm{C} / \mathrm{kg}$ and $|v|=100 \mathrm{~km} \mathrm{~s}^{-1}$ ) is about $2 \mathrm{AU}$, which is much larger than the gyroradius of oxygen PUIs $\left(2.2 \cdot 10^{-3} \mathrm{AU}\right.$, or $\sim 5.5 R_{S} ; 1 R_{S}=60,268 \mathrm{~km}$ is the equatorial radius of Saturn) as well as the size of Saturn's 


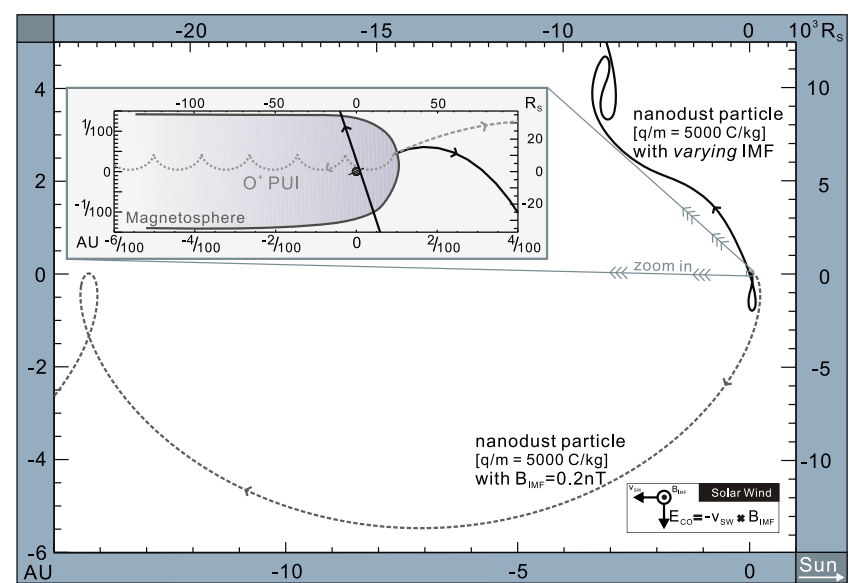

Figure 1. Trajectories of an $\mathrm{O}^{+}$PUI (gray dotted line) and charged nanoparticles (solid and dashed lines) in the solar wind near Saturn. The magnetosphere of Saturn is also shown schematically. The gray, dashed line represents the trajectory of a nanodust particle $(q / m=5000 \mathrm{C} / \mathrm{kg})$ under constant IMF condition. The black solid line is the trajectory of the same particle but under varying IMF conditions as described in section 4 and Figure 3. Notice that this particle reenters the magnetosphere of Saturn from the south after experiencing northward acceleration within the solar wind compression.

magnetosphere $(<0.1$ AU). Figure 1 illustrates the trajectories of a PUI and charged nanoparticles including the $\mathbf{E} \times \mathbf{B}$ drift. It shows that the pickup process of atomic ions takes place in a relatively small spatial scale while the pickup region of stream particles is a few $\mathrm{AU}$ around their source planet.

[6] As for the temporal scale, the gyroperiod of an aforementioned nanoparticle under a constant IMF condition is $\sim 70$ days, much longer than oxygen PUIs' $(\sim 1 \mathrm{~h})$ and the solar rotation period $(\sim 27$ days $)$. The implication of such a long gyroperiod of charged nanoparticles is a complex dynamical evolution as shown in Figure 1, since they most likely experience varying IMF conditions during the pickup process.

[7] Cassini's Saturn approach took place during the declining phase of the solar cycle. The IMF during this period recurrently shows a two-sector structure and is organized as rarefaction and compression regions associated with corotating interaction regions (CIRs) [Jackman et al., 2004]. The field strength ranges from $0.1 \mathrm{nT}$ in rarefaction regions to $>1 \mathrm{nT}$ in solar wind compressions. The IMF configuration generally follows the Parker spiral model, i.e., dominated by the component tangential to the solar wind flow direction [Jackman et al., 2008]. The heliospheric current sheet (HCS), which marks the boundary between IMF sectors, is mostly embedded within solar wind compressions [Jackman et al., 2004]. An idealized IMF time sequence as seen by a stationary probe near Saturn thus consists of a solar wind rarefaction region followed by a sharp increase in IMF strength, indicating the arrival of the solar wind compression. Within the compression, the probe encounters the HCS, marked by a $180^{\circ}$ field rotation, and enters another sector. Afterward, the field strength gradually reduces to the rarefaction region level. Such a sequence takes about half a solar rotation period and then repeats with an opposite IMF polarity.

[8] The major influence of such an IMF profile on the nanodust pickup process is that not only the magnitude but also the direction of $\mathbf{E}_{\mathbf{c}}$ varies with time. Hence, stream particles ejected at different IMF phases will follow completely different dynamical evolution paths. This influence is clearly reflected by the in situ dust measurements in interplanetary space.

[9] In situ dust measurement at a given time provides a snapshot of the dust dynamical evolution at the spacecraft location. Although it only covers a small part of the nanodust pickup process, it shows the immediate dynamical reaction of nanoparticles to the IMF condition. For example, in solar wind rarefaction regions, the IMF and $\mathbf{E}_{\mathbf{c}}$ are relatively weak. The dynamics of nanoparticles ejected during this time are not affected significantly by the solar wind. Their trajectories thus remain relatively unperturbed in the vicinity of their source planet.

[10] In solar wind compressions, however, $\mathbf{E}_{\mathbf{c}}$ could be 10 times higher than that in rarefaction regions, which results in significant stream particle acceleration within a few hours. Given that the IMF at Saturn is governed by the tangential component, $\mathbf{E}_{\mathbf{c}}$ predominantly points either northward or southward, depending on the IMF sector, with respect to the ecliptic plane. Upon entering a solar wind compression, the nanodust dynamics will quickly be overwhelmed by this rapidly changing acceleration that drives the nanoparticles away from the ecliptic plane. This leads to noticeable changes in the directionality, flux, and signal strength in the in situ nanodust measurements. The changes are found to be highly correlated with IMF variations and form a recurrent pattern in dust measurements within the solar wind in the vicinity of Saturn as well as Jupiter [Grün et al., 1993; Hamilton and Burns, 1993; Kempf et al., 2005; Krüger et al., 2006; Hsu et al., 2010; Flandes et al., 2011; Hsu et al., 2011a].

[11] Interestingly, similar nanodust detection patterns are also registered when the spacecraft is inside the magnetosphere of Saturn. Figure 2 shows the nanodust measurements carried out by the Cassini Cosmic Dust Analyzer (CDA) [Srama et al., 2004] during 2006 day of year (DOY) 020-090 (20 January to 31 March). The time and directionality of each nanoparticle detection are shown by the triangle/square symbols color coded with the impact charge yield. The impact charge yield is the amount of plasma produced from the dust-CDA target impact. It exhibits a sensitive dependence on the particle speed [Göller and Grün, 1989].

[12] In Figure 2, one can identify two dust components according to the impact charge and directionality - the first one is composed of weaker signals registered once CDA points toward Saturn and the ring plane (i.e., small CDASaturn and CDA-RP angles). This component most likely consists of nanoparticles ejecting from the system, whose dynamics are not yet influenced by the IMF. The second component is characterized by higher impact charges and specific directionality $\left(>45^{\circ}\right.$ away from Saturn and the ring plane direction), which are similar to the measurements in interplanetary space during strong IMF periods. Unlike the weak but consistent Saturn line-of-sight component, the energetic impacts appear in recurrent swarms that occur about every solar rotation (sometimes half of the solar 


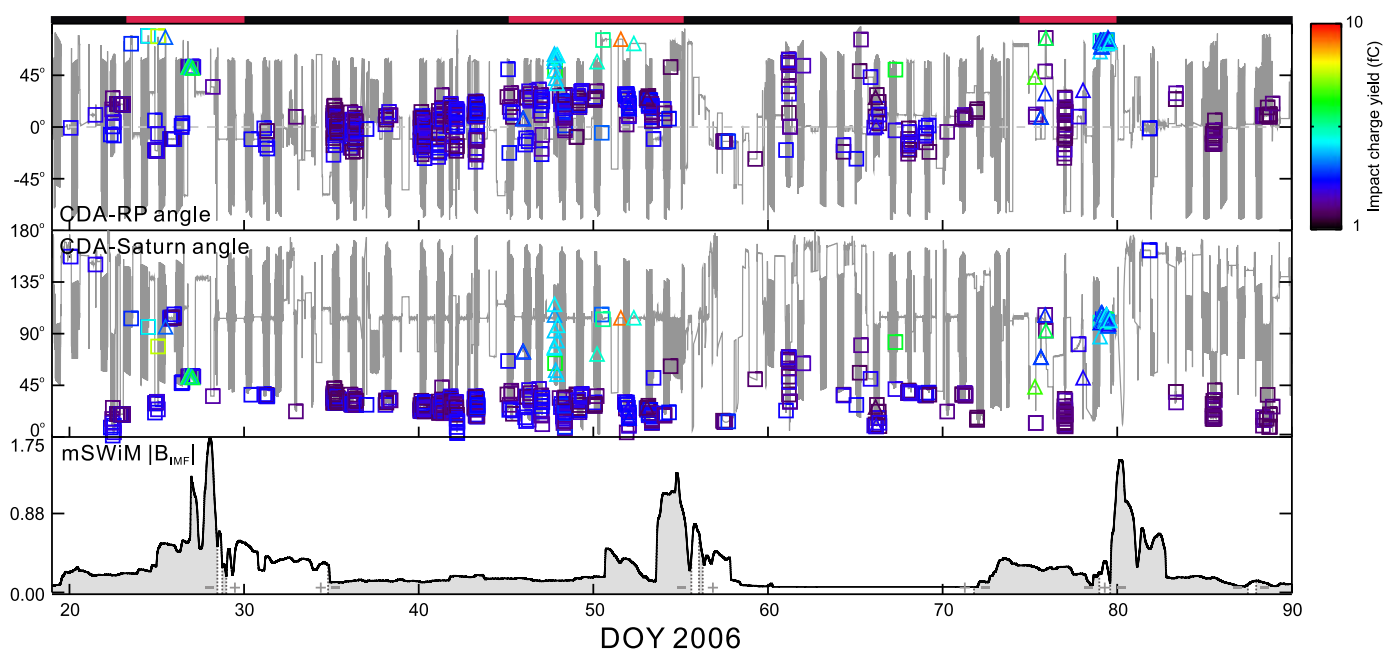

Figure 2. Nanodust measurements and the simulated IMF strength at Saturn during 2006 days 20 to 90. CDA nanodust measurements shown at the top two panels are carried out when the spacecraft is in the magnetosphere. Individual nanoparticle detections are marked by the square/triangle symbols and color coded with the impact charge yield. Their directionality along with the CDA pointing profile is expressed by two angles-CDA-Saturn angle and CDA-RP (Ring Plane) angle, which are the angular separations between the instrument boresight and the Saturn line of sight and Saturn's ring plane, respectively. Two impact components can be identified based on the dust directionality and the impact charge yield, as described in section 2. Periods with higher impact charge component are indicated by the red bars on the top. The bottom panel shows the IMF strength and polarity at Saturn derived from the Michigan Solar Wind Model. The field polarity is marked by the + (toward sector) and - (away sector, also shaded in gray color) signs.

rotation period, not shown here). The CDA-RP angle of these detections is $>+45^{\circ}$, suggesting that they were moving southward toward the Saturnian system. Although no direct solar wind measurements are available during this period, this detection pattern resembles dust measurements in the solar wind compressions. This observation strongly suggests that the energetic dust component (seen inside the magnetosphere) forms due to the same mechanism: the nanodustIMF interactions.

\section{Comparison With the Michigan Solar Wind Model}

[13] Since there are no direct solar wind measurements near Saturn during this period, an alternative method to examine the proposed scenario is to use results from the solar wind propagation model. The Michigan Solar Wind Model (mSWiM) [Zieger et al., 2008] adopts measurements near Earth as inputs and derives the solar wind properties as it propagates outward using a one-dimensional (1-D) magnetohydrodynamic code. One limitation of this method is that the modeling results become less precise with increasing longitudinal separation between the Earth, where the input data are acquired, and the target (in this case, Saturn). Fortunately, the above observation takes place around the "apparent opposition" (in 2006 Day 56), defined as the best alignment condition between the Earth and Saturn in terms of the solar wind propagation [Zieger et al., 2008]. This model thus provides us with predicted solar wind information at Saturn with the best possible temporal precision.

[14] The bottom panel of Figure 2 shows the IMF strength at Saturn calculated from the Michigan Solar Wind Model. Comparing with CDA measurements, the occurrence of the energetic nanodust component coincides well with periods when the propagated field is strong (2006 DOY 23-30,
45-55, and 74-80). Moreover, the velocity vector of the energetic dust component and the direction of $\mathbf{E}_{\mathbf{c}}$ derived from the mSWiM IMF polarity both point southward, indicating that the dynamical properties of these nanoparticles most likely result from their recent acceleration in the solar wind.

\section{Nanodust Dynamics Modeling}

[15] To further examine the proposed scenario, we develop a numerical model that employs a "toy IMF model" to simulate the dynamical evolution of ejected nanoparticles under varying IMF conditions. Our IMF model is a 1-D description of four time-varying parameters - the solar wind speed $\left(v_{\mathrm{sw}}\right)$, the IMF vector $\mathbf{B}_{\mathrm{IMF}}$, the plasma density $\left(N_{\mathrm{sw}}\right)$, and the plasma temperature $\left(T_{\mathrm{sw}}\right)$. The first two parameters directly affect the dust dynamics, and the latter two are used for the dust charging calculation. These parameter profiles follow the aforementioned IMF sequence and are shown in Figure 3. Despite its simplicity, our IMF model replicates the most important features of CIRs at Saturn's orbit during the declining solar cycle. It may not be able to reproduce specific measurements but should be fairly representative in terms of IMF-nanodust interactions.

[16] In order to examine the recurrent stream particle activity, the trajectory of each test particle is traced for one complete solar rotation period to account for the cumulated dynamical influence from the time-varying IMF. The simulation takes into account the electromagnetic forces as well as the gravity from the Sun and Saturn, despite that the gravitational forces are weaker by 3 orders of magnitude or more [Zook et al., 1996]. Dust charging is also included, though here it has only minor effects. Test particles with 2 to $8 \mathrm{~nm}$ radii are launched from Saturn with charge-to-mass ratio and speed following the relation $|v|=1.34 \times(\mathrm{q} / \mathrm{m})^{0.5}$ 


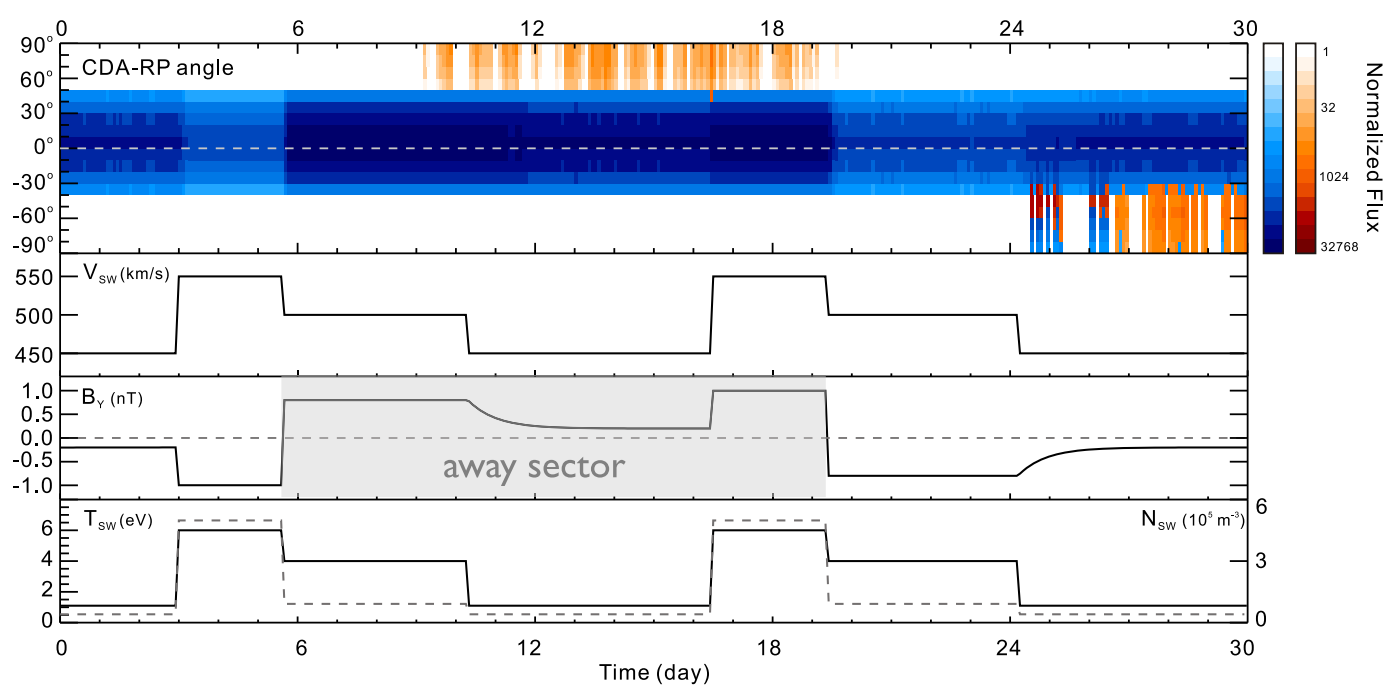

Figure 3. The dust dynamics simulation result and the adopted toy IMF model. The top panel shows the simulated stream particle detection pattern as measured by a spacecraft in Saturn's equatorial plane. The simulated particle flux is binned with respect to the CDA-RP angle as a function of time. Two color systems are applied based on the average impact charge yield in each bin (blue: $<2 \mathrm{fC}$; orange: $\geq 2 \mathrm{fC}$ ). Based on the directionality and the impact charge, two dust components are clearly distinguishable from the simulation. Particles with lower impact charges are continuously registered from the equatorial direction. Stronger impacts are predominately registered from the north and south of the ring plane, indicating the dynamical influence of $\mathbf{E}_{\mathbf{c}}$ in the solar wind. The IMF model adopted in the simulation is shown in the lower three panelsthe solar wind speed $\left(v_{\mathrm{sw}}\right)$, the tangential component of IMF $\left(B_{Y}\right)$, and the solar wind plasma density $\left(N_{\mathrm{sw}}\right.$; gray dashed line) and electron temperature $\left(T_{\mathrm{sw}} ;\right.$ solid line).

( $v$ in $\mathrm{km} \mathrm{s}^{-1}$ and $q / m$ in $\mathrm{C} / \mathrm{kg}$ ) derived by Hsu et al. [2011b]. The velocity vectors of particles whose trajectories intersect with the defined "magnetosphere" (the volume within $\pm 40 R_{S} \times \pm 50 R_{S}$ along and $20 R_{S}$ above and below the ring plane) are recorded and regarded as nanodust measurements inside the magnetosphere. Note that the magnetic field of Saturn is not considered in the calculation as the size of the magnetosphere, compared to the stream particle pickup region, is too small to have noteworthy effects on nanoparticles traversing through with a speed $\gg 100 \mathrm{~km} \mathrm{~s}^{-1}$. The simulated detection pattern is achieved by convolving dust directionality with the CDA's sensitivity area, assuming that the observation is made within Saturn's ring plane with homogenous angular coverage.

[17] The simulation result from about 2 million test particles is shown in the top panel of Figure 3. The simulated detection pattern is expressed by the CDA-RP angle and does show two dust components - the one registered from the ring plane direction corresponds to the slower particles that are just escaping the Saturnian system (blue color). Another component detected at large CDA-RP angles corresponds to the IMF-modified, fast dust component as indicated by their higher charge yield (mostly orange color). Since the simulation starts with only the slow component, this result clearly indicates that both nanodust components are particles with the same origin but different dynamical histories, resulting from the dynamical influence under changing solar wind conditions.

[18] As mentioned, the directionality of registered fast particles directly reflects the directions of $\mathbf{E}_{\mathbf{c}}$ and IMF, because $\mathbf{E}_{\mathbf{c}}$ is mainly responsible for the nanodust acceleration in the solar wind. As shown in Figure 3, the particle directionality reliably reflects the IMF polarity throughout the simulation. The flux ratio between the two simulated components qualitatively agrees with the observation; i.e., the flux of slow particles is much higher than the fast ones. This is most likely because of the scattering caused by the IMF, which behaves similarly to a giant mass spectrometer that scatters nanoparticles with various charge-to-mass ratios during the pickup process.

\section{Discussion and Summary}

[19] Using two independent approaches, we show that the recurrent energetic nanodust component observed inside the magnetosphere consists of nanoparticles that are originally ejected and then reenter the magnetosphere of Saturn at certain occasions due to the dynamical influence from the time-varying solar wind conditions. With continuous dust measurements, information about the IMF can be obtained from inside the magnetosphere of Saturn.

[20] The two key elements for sending ejected nanoparticles back to the magnetosphere are as follows: (1) the IMF strength enhancement and (2) the IMF direction reversal. These factors are closely related to the type of IMF information that the nanodust measurements can provide.

[21] Nanodust measurements can provide a rough estimate on the arrival time of solar wind compressions. However, there will be a time lag between the arrival of solar wind compressions at Saturn and the beginning of energetic nanoparticle detection because nanoparticles can only be sent back to the magnetosphere after experiencing the field reversal (i.e., HCS crossing). The actual time difference depends on several factors, such as the geometry of the CIR shock front and the large-scale IMF structure.

[22] In our simulation, the detection of the fast component begins at day 9 , about 6 days later than the arrival of the solar wind compression. This time difference is much larger 
than that suggested from the observation in the solar wind, which is about 2 days [Hsu et al., 2010, 2011a]. This may be due to the simplicity of our "toy IMF model," as it may only partially reproduce the long-term, cumulative effects on nanodust dynamics as we ignore the latitudinal variation of solar wind.

[23] Nonetheless, despite this temporal inaccuracy, nanodust observation is an independent indicator for the arrival of solar wind compression at Saturn, which is still useful for studying the internal magnetospheric processes during quiet solar wind conditions [e.g., Gérard et al., 2006] or for exploring the effects of solar wind on Saturn kilometric radiation [e.g., Badman et al., 2008] and on the magnetospheric dynamics [e.g., Jackman et al., 2010].

[24] Another solar wind property that can be derived more reliably is the IMF direction, as demonstrated by our dust dynamics model. Considering the two-sector IMF structure, the data shown in Figure 2 suggest that Saturn was located in the "away" IMF sector (i.e., the IMF direction is close to dawn to dusk) during 2006 DOY 23-30, 45-55, and 74-80.

[25] One limiting factor of the proposed method stems from the finite angular coverage. The instrument has a finite field of view, and its pointing profile is complicated by the operation of the three-axis stabilized Cassini spacecraft. A wider angular coverage (e.g., spacecraft rolls) is more favorable for the purpose of nanodust/IMF monitoring.

[26] A more complete survey including field direction, solar wind compression arrival time, and the corresponding error estimation based on nanodust measurements during the Cassini prime mission (2004-2008) is in preparation. Future improvements of the proposed method include (1) exploring the reaction of nanodust dynamics under different IMF geometries and (2) application to conditions at Jupiter and other potential nanodust-host planets.

[27] Acknowledgments. We acknowledge support from the Cassini project. We thank Tamas Gombosi for the fruitful discussion. H.-W. Hsu thanks Paige Northway for her assistance in improving the readability of the manuscript.

[28] The Editor thanks two anonymous reviewers for their assistance in evaluating this paper.

\section{References}

Badman, S. V., S. W. H. Cowley, L. Lamy, B. Cecconi, and P. Zarka (2008), Relationship between solar wind corotating interaction regions and the phasing and intensity of Saturn kilometric radiation bursts, Ann. Geophys., 26, 3641-3651, doi:10.5194/angeo-26-3641-2008.

Flandes, A., H. Krüger, D. P. Hamilton, J. F. Valdés-Galicia, L. Spilker, and R. Caballero (2011), Magnetic field modulated dust streams from Jupiter in interplanetary space, Planet. Space Sci., 59, 1455-1471, doi:10.1016/j.pss.2011.05.014.
Gérard, J.-C., et al. (2006), Saturn's auroral morphology and activity during quiet magnetospheric conditions, J. Geophys. Res., 111, A12210, doi:10.1029/2006JA011965.

Göller, J. R., and E. Grün (1989), Calibration of the Galileo/Ulysses dust detectors with different projectile materials and at varying impact angles, Planet. Space Sci., 37, 1197-1206.

Grün, E., et al. (1993), Discovery of Jovian dust streams and interstellar grains by the Ulysses spacecraft, Nature, 362, 428-430.

Hill, M. E., D. C. Hamilton, G. Gloeckler, S. M. Krimigis, and D. G. Mitchell (2004), Near-Saturn solar wind speeds determined from MIMI/CHEMS measurements of pickup ion spectra at the Cassini spacecraft, Eos Trans. AGU, 85(47), Fall Meet. Suppl., Abstract P51A-1412.

Hamilton, D., and J. Burns (1993), Ejection of dust from Jupiter's gossamer ring, Nature, 364, 695-699.

Horányi, M. (2000), Dust streams from Jupiter and Saturn, Phys. Plasmas, 7(10), 3847-3850.

Horányi, M., E. Grün, and A. Heck (1997), Modeling the Galileo dust measurements at Jupiter, Geophys. Res. Lett., 24, 2175, doi:10.1029/ 97GL01539.

Hsu, H.-W., S. Kempf, and C. M. Jackman (2010), Observation of Saturnian stream particles in the interplanetary space, Icarus, 206, 653-661, doi:10.1016/j.icarus.2009.06.033.

Hsu, H.-W., S. Kempf, F. Postberg, M. Trieloff, M. E. Burton, M. Roy, G. Moragas-Klostermeyer, and R. Srama (2011a), Cassini dust stream particle measurements during the first three orbits at Saturn, J. Geophys. Res., 116, A08213, doi:10.1029/2010JA015959.

Hsu, H.-W., F. Postberg, S. Kempf, M. Trieloff, M. Burton, M. Roy, G. Moragas-Klostermeyer, and R. Srama (2011b), Stream particles as the probe of the dust-plasma-magnetosphere interaction at Saturn, J. Geophys. Res., 116, A09215, doi:10.1029/2011JA016488.

Hsu, H.-W., H. Krüger, and F. Postberg (2012), Dynamics, composition, and origin of Jovian and Saturnian dust-stream particles, in Nanodust in the Solar System: Discoveries and Interpretations, Astrophys. Space Sci. Libr., vol. 385, edited by I. Mann, N. Meyer-Vernet, and A. Czechowski, pp. 77-177, Springer, New York, doi:10.1007/978-3-642-27543-2 5.

Jackman, C. M., N. Achilleos, E. J. Bunce, S. W. H. Cowley, M. K. Dougherty, G. H. Jones, S. E. Milan, and E. J. Smith (2004), Interplanetary magnetic field at $\sim 9 \mathrm{AU}$ during the declining phase of the solar cycle and its implications for Saturn's magnetospheric dynamics, J. Geophys. Res., 109, A11203, doi:10.1029/2004JA010614.

Jackman, C. M., R. J. Forsyth, and M. K. Dougherty (2008), The overall configuration of the interplanetary magnetic field upstream of Saturn as revealed by Cassini observations, J. Geophys. Res., 113, A08114, doi:10.1029/2008JA013083.

Jackman, C. M., C. S. Arridge, J. A. Slavin, S. E. Milan, L. Lamy, M. K. Dougherty, and A. J. Coates (2010), In situ observations of the effect of a solar wind compression on Saturn's magnetotail, J. Geophys. Res., 115, A10240, doi:10.1029/2010JA015312.

Kempf, S., R. Srama, M. Horányi, M. Burton, S. Helfert, G. MoragasKlostermeyer, M. Roy, and E. Grün (2005), High-velocity streams of dust originating from Saturn, Nature, 433, 289-291.

Krüger, H., A. L. Graps, D. P. Hamilton, A. Flandes, R. J. Forsyth, M Horányi, and E. Grün (2006), Ulysses Jovian latitude scan of highvelocity dust streams originating from the Jovian system, Planet. Space Sci., 54, 919-931, doi:10.1016/j.pss.2006.05.010.

Srama, R., et al. (2004), The Cassini Cosmic Dust Analyzer, Space Sci. Rev., 114, 465-518, doi:10.1007/s11214-004-1435-z.

Zieger, B., and K. C. Hansen (2008), Statistical validation of a solar wind propagation model from 1 to $10 \mathrm{AU}$, J. Geophys. Res., 113, A08107, doi:10.1029/2008JA013046.

Zook, H., E. Grün, M. Baguhl, D. Hamilton, G. Linkert, J.-C. Liou, R. Forsyth, and J. Phillips (1996), Solar wind magnetic field bending of Jovian dust trajectories, Science, 274, 1501-1503. 